\title{
LOAD BALANCING APPROACH TO ENHANCE THE PERFORMANCE IN CLOUD COMPUTING
}

\author{
Noof Alarifi and Iehab Alrassan ${ }^{1}$ \\ King Saud University, Department of Computer Science, Riyadh, Saudi Arabia \\ ${ }^{l} \mathrm{Dr}$.
}

\begin{abstract}
Virtualization technologies are being adopted and broadly utilized in many fields and at different levels. In cloud computing, achieving load balancing across large distributed virtual machines is considered as a complex optimization problem with an essential importance in cloud computing systems and data centers. The overloading or underloading of tasks on Virtual machines (VMs) may cause multiple issues in the cloud system like longer execution time, machine failure, high power consumption, etc. Therefore, load balancing mechanism is an important aspect in cloud computing that assists in overcoming different performance issues. This paper discusses a new approach that combines the advantages of Round robin algorithm with a threshold technique. The proposed design enhances scheduling policies to overcome the resource utilization problem without compromising other performance measures like makespan and completion rate of the tasks. The proposed system design will be expecting providing better results compared to the original round robin as it takes into consideration the dynamic state of the system.
\end{abstract}

\section{KEYWORDS}

Cloud Computing, Load Balancing, Round Robin, Virtual Machine

\section{INTRODUCTION}

Within the recent decade, major innovations in the field of network technology have emerged, that potentially add more convenience to daily life practices not only on an enterprise level but on an individual level as well. Cloud computing technology has witnessed significant advances in its implementation and becomes widely adopted by both private and public sectors since it provides different resources over the Internet. Cloud computing allows sharing hardware and software resources like networks, servers, storage, software, applications, etc. all over the Internet since it is an Internet-based computing model (Mishra, Sahoo \& Parida, 2019).

In cloud systems different nodes might be assigned with uneven loads of tasks where some nodes are overloaded and other nodes underloaded which may affect the overall performance and cause multiple issues like longer execution time, machine failure, high power consumption, etc. Therefore, load balancing mechanism is an essential aspect in cloud computing which is concerned with detecting the nodes that are overloaded or underloaded with tasks and balance the load among them.

Using an efficient load balancing mechanism is an important aspect in cloud computing that assists in overcoming different performance issues. One of the most significant aspect of cloud computing is the virtualization technology where there is a several virtual machines that might run different operating systems on one physical host. Virtualization is achieved through the help of hypervisor; also called virtual machine monitor (VMM) which can be placed over the hardware directly or upon the operating system of the host machine. The virtual machine migration is the process of transferring a virtual machine to another physical machine in order to improve the resource utilization in case of the physical machine was overloaded. The task migration is the process of transferring a task from one virtual machine to another virtual machine either on the same physical machine or to a virtual machine on another physical machine. Virtual machine migration and task migration play an important role in load balancing in cloud computing (Shirvani, Rahmani \& Sahafi, 2018). In this paper, a proposal for a new approach that combines the advantages of Round robin algorithm with a threshold technique. The proposed design expects to enhance scheduling policies to overcome the resource utilization problem without compromising other performance measures: makespan and completion rate. 
The reminder of this paper organized as follows: section 2 includes a detailed background about the cloud computing architecture, and load balancing algorithms. Section 3 presents a review of the existing works on load balancing in cloud systems. Section 4 includes the proposed approach for achieving the load balance among different targeted virtual machines. Section 5 is the conclusion of the paper.

\section{CLOUD COMPUTING ARCHITECTURE}

The layer of hardware comprises of hardware resources that include network bandwidth, main memory, processor, and secondary storage. Hypervisor such as Denali, VMWare, Xen, UML, or Virtual Machine Monitor will act as a crossing point between the Virtual machines and the guest operating system. This Virtual Machine supports numerous operating systems that simultaneously execute applications in only one hardware platform. Various heterogeneous applications run on every virtual machine. \{VM1; VM2; VM3; ...; VMn \} be the set of VMs used in the cloud hosts (Mishra, Sahoo \& Parida, 2019). Figure 1 shows the architecture of cloud computing.

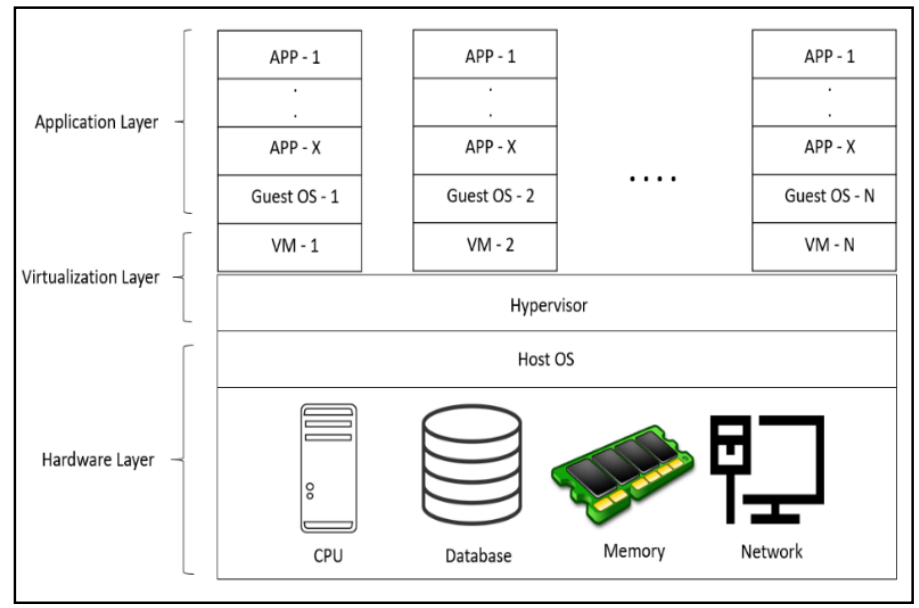

Figure 1. Architecture of Cloud Computing

The data center of the cloud consists of fixed number of various physical hosts. The identification of every host is through its lists of processing elements, bandwidth, identification number, memory size, and speed for processing in terms of Million instructions per second (MIPS) (Mishra, Sahoo \& Parida, 2019).

Cloud computing is concerned with services, software, and hardware provisioning from third parties via the use of a network. Any cloud computing system contains substantial components like datacenter, distributed servers, as well as clients (Ray \& De Sarkar, 2012):

a) Datacenter: is a group of servers hosting various applications. The end-user links to the datacenter to subscribe to various applications. It may be on a big remoteness from the clients' position.

b) Distributed servers: are part of a cloud that are accessible through the internet. The server hosts various applications, the users may feel that they are using these applications from their own computers.

c) Client: the end-users communicate with the clouds for information management associated with the cloud.

\section{LOAD BALANCING ALGORITHMS}

Based on the current state of the system, load balancing algorithms can be divided into two categories (Ramana \& Ponnavaikko, 2019); Static algorithms where decisions on the load balancing doesn't depend on the current state of the system. It needs prior information about the average behavior of the system. Second category is dynamic algorithms where decisions on load balancing are made depending on the current state of the system, 
which means that more benefits can be achieved compared to the static policies. It is more complex since no prior information is used.

Brief review of the current algorithms of load balancing are presented as follows:

- Round Robin

The processes in this algorithm are divided amongst all processors. In a round robin order, every task is allocated to a processor. The workload allocation amongst the processors are the same but the processing time of each job is different, one job may take longer execution time than the others, at any time some nodes may be loaded heavily, and others continue to be idle or lightly loaded. Round robin is a static algorithm, it does not need inter process communication which results in a less system overhead (Eager et al., 1986).

- Central queuing

Central queuing algorithm is a dynamic allocation algorithm. Each new arriving activity is inserted into the queue. Once the queue manager receives the requests for an activity, it deletes the first activity from the queue and directs it to the requester. If there is no activity ready in the queue, the request is buffered till a new activity is available. In case a new activity comes to the queue whereas there are requests that have not been answered the first request is detached from the queue and a new activity is allocated to it (Elngomi \& Khanfar, 2016).

- Min-Min

The primary procedure of this technique is to select the task with the smallest size as well as a virtual machine with the least resource or capacity. Once the task allocation to a virtual machine is done, the task is deleted from the queue and carry on with the allocation of all tasks that are unassigned. This algorithm is appropriate for distributed systems that are only small-scale (Mishra, Sahoo \& Parida, 2019).

- Min-Max algorithm

It is the same as the Min-Min. In the cloud computing, the primary procedure of Max-Min is to assign the task that is larger in size to a virtual machine with the least resource or capacity. Once the tasks distribution to a virtual machine is done, it is deleted from the queue then continue with the rest of unallocated tasks. This algorithm is appropriate for distributed systems of small-scale only (Mishra, Sahoo \& Parida, 2019).

\section{PREVIOUS WORK}

Achieving load balancing across large distributed servers is considered a complex optimization problem with an essential importance in cloud computing systems and data centers. Existing schedulers often incur a high communication overhead when collecting the data required to make scheduling decisions, hence delaying job requests on their way to the executing servers.

Many approaches have been developed to overcome the problem of uneven load distribution among nodes in cloud computing. An approach for load balancing is proposed in (Sharma, Luhach \& Sinha, 2016) which is based on Bat Algorithm where it is supposed to find the optimal host as well as VM for any incoming task. The BAT algorithm is enforced by a load balancer in case there is a task that has arrived in the job pool where the algorithm will select a server that matches the incoming task. The main factor considered when applying the BAT algorithm is the type of the task and the required resources for the excellent assignment execution. The proposed BAT algorithm was evaluated by comparing it with fuzzy and GSO and round-robin.

In (Li et al., 2011), The authors introduced a scheduling approach based on Load Balancing Ant Colony Optimization (LBACO) algorithm. The main goal was trying to balance the system load and reducing the makespan which is the total time needed to complete all tasks submitted to the system. For the evaluation process, the results of the proposed LBACO algorithm was compared to FCFS (First Come First Serve) and the basic ACO (Ant Colony Optimization) using simulations.

The work in (Maheshwari \& Gupta, 2019) intends to measure the optimization of cloud computing performance. Hence, there are two significant approaches to scheduling. There are two primary scheduling techniques applied with the help of CloudSim simulator: Round Robin and the first come first served. The round-robin approach is a time-shared concept where a fixed amount of time is assigned to a given job for all resources. The FCFS approach works depending on the space shared manner whereby tasks are allocated depending on their appearance in the array. The two techniques' performance are compared and measured. The comparison of the performance calculations is done by the use of a simulation trace where the average processing time, average waiting time and utilization of the CPU computations are considered. The round-robin approach is proven to be more efficient in all criterions. 
As virtualization technologies are being adopted and broadly utilized in many fields and at different levels, it has a huge significance and there must be an efficient virtual machine load balancing and migration schemes to serve as a tool for managing cloud resources and achieve the primary goals like maintaining the load balance, reducing the failure possibilities, resource utilization and so on.

\section{METHODOLOGY}

In this paper, we propose a method that combines the advantages of Round robin with a threshold technique. Thus, the proposed scheduler enhances scheduling policies to overcome the resource utilization problem and to reduce the makespan of the system. An overview of the environment and its components is presented in the next subsection.

\subsection{System Main Components}

A typical environment for modeling cloud systems usually involves datacenters, set of host machines and a set of virtual machines.

- Datacenter: is centralized place that involves numerous servers where computing and networking is taking place in the cloud.

- Host: Host machines are connected to the datacenter. Hosts involves set of virtual machines.

- VM: is virtual machine that is applied on a physical machine. Every virtual machine may run different OS than the physical machine and have their own resources.

There are an $n$ input of tasks (T) from 1 to $n\left(T_{1}, T_{2}, \ldots, T_{n}\right)$, and $N$ numbers of virtual machines (VM) from 1 to $N\left(V M_{1}, V M_{2}, \ldots, V M_{N}\right)$. Load balancer is essentially based on two characteristics. First, assign load to the best candidate node, and second, migration of load from heavily loaded VMs to lightly loaded VMs.

\subsection{Proposed Work}

Round Robin is a simple scheduling algorithm as it distributes the load evenly among the existing VMs in a round robin style, which results in less system overhead, however, it doesn't take into consideration the different amount of resources that are allocated to different VMs, which in return might cause some lacking in other performance metrics like makespan, resource utilization and completion rate of the tasks. Figure 4 shows the distribution of tasks across virtual machines using round robin in one host.

Round robin can be adequate in small systems when all VMs have the same resource configurations. However, in this approach we assume that all VMs have different resources. Thus, after the distribution of the first load in a round robin style, the utilization of the resources is calculated, then we are going to use the weighted round robin algorithm, where each VM is assigned with a weight that indicates its amount of available resources and based on this weight the load will be distributed in a round robin style. This assures the even distribution of load. However, it doesn't necessarily improve the makespan or completion rate as the load might be so heavy on some or all existing VMs.

Therefore, a threshold is defined, when this threshold is exceeded, then the tasks should be migrated either to another VMs in the same host, or to a VM in another host. First, the VMs in the same host will be checked out as some tasks might have finished their execution, if not then, the tasks should be migrated to another host.

In case all the VMs on the same host are overloaded, the queue of the incoming tasks should stop assigning these tasks to the VMs until they get lighter in load or migrate these tasks to another VM on another host. The host will be picked up according to its load-level state, which is kept and updated in a table in the datacenter. The state of the host is represented by the number of the idle VMs divided by the total number of VMs on that host which will provide a percentage that represent the state of the host. The states of different hosts in the datacenter will be arranged in ascending order where the lightest-loaded host is placed on the top, and this order is updated in every time step. 


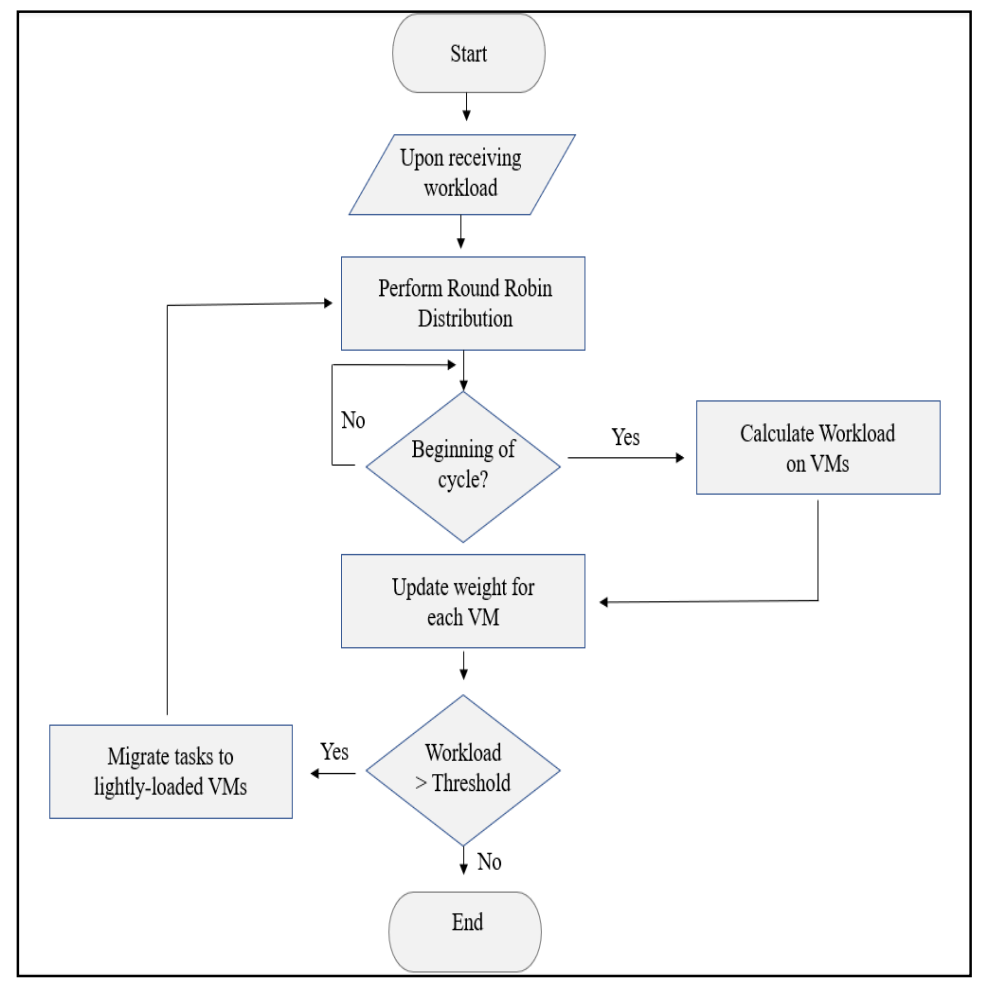

Figure 2. Flowchart of the proposed work in one host

\subsection{Evaluation}

There are different performance metrics that can be affected from the mapping of tasks to the virtual machines. The following performance metrics are considered in analyzing the performance of the proposed load balancing approach:

- Completion Rate:

The rate of the completed jobs will be calculated as the ratio between the number of requested jobs and the number of completed jobs:

- Makespan:

$$
\text { Completion Rate }=\frac{\text { Number of Requested Jobs }}{\text { Number of Completed Jobs }}
$$

This is the amount of time spent between the start and end of all tasks executed by the system. In this case, makespan is the total or maximum time taken by the host to run all tasks. Makespan (MS) is the maximum of $E T_{j}$, calculated as follows (Mishra, Sahoo \& Parida, 2019):

$$
M S=\operatorname{Max} \cdot\left[E T_{j}\right]_{j=1}^{n}
$$

First the Expected Time to Compute is calculated as: $E T C_{i j}=\frac{L_{i}}{P_{j}}$, where $L_{i}$ is the length of the task in terms of Million instructions (MI), and $P_{j}$ is the processing speed of the VM in terms of MIPS. Then the Execution Time of VM is calculated as:

$$
\begin{gathered}
X_{i j}= \begin{cases}1 & \text { if } T_{i} \text { is allocated to } V M_{j} \\
0 & \text { if } T_{i} \text { is not allocated to } V M_{j}\end{cases} \\
E T_{j}=\sum_{i=1}^{n} X_{i j} \times E T C_{i j}
\end{gathered}
$$

We assume that the metrics of the proposed system will provide better results compared to the original round robin as it takes into consideration the dynamic state of the system. 


\section{CONCLUSION}

Virtualization technology is one of the most significant aspect of cloud computing where there is a several virtual machines that might run different operating system on one physical host. The critical issue in cloud computing is the balancing of loads across these VMs and achieving an excellent utilization of resources. The workload must be managed equally across all VMs. There is a need for the development of a new approach for load balancing that overcomes the existing methods drawbacks. Scheduling is an aspect that should be improved to obtain an efficient performance. The objective of scheduling is mapping tasks to resources to optimize one or several objectives. In cloud computing, scheduling belongs to NP-hard problem category as a result of wide solution space. There is no existence of an algorithm that can find the optimal solution within polynomial time. It is recommended to find a suboptimal solution technique for achieving solutions within a reasonable time.

In this paper, we proposed an approach that combines the advantages of Round robin with a threshold technique. Thus, the proposed scheduler expects to enhance scheduling policies to overcome the resource utilization problem and to reduce the makespan of the system. To evaluate the proposed work, the results will be discussed in the context of makespan and completion rate metrics and the performance will be compared to the original round robin. For future work, we will consider the priority of tasks and execute the tasks with higher priority first without preemption and predict the incoming tasks by utilizing a prediction technique.

\section{REFERENCES}

Arunarani, A.R., Manjula, D. and Sugumaran, V., 2019. Task scheduling techniques in cloud computing: A literature survey. Future Generation Computer Systems, 91, pp.407-415.

Eager, D.L., Lazowska, E.D. and Zahorjan, J., 1986. A comparison of receiver-initiated and sender-initiated adaptive load sharing. Performance evaluation, 6(1), pp.53-68.

Elngomi, Z.M. and Khanfar, K., 2016. A Comparative Study of Load Balancing Algorithms: A Review Paper. International Journal of Computer Science and Mobile Computing, 5(6), pp.448-458.

Hota, A., Mohapatra, S. and Mohanty, S., 2019. Survey of different load balancing approach-based algorithms in cloud computing: a comprehensive review. In Computational Intelligence in Data Mining (pp. 99-110). Springer, Singapore.

Maheshwari, K. and Gupta, V.K., 2019. Load Balancing in VM in Cloud Computing Using CloudSim. Available at SSRN 3372902.

Mell, P. and Grance, T., 2011. The NIST definition of cloud computing.

Mishra, S.K., Sahoo, B. and Parida, P.P., 2018. Load balancing in cloud computing: A big picture. Journal of King Saud University-Computer and Information Sciences.

Ray, S. and De Sarkar, A., 2012. Execution analysis of load balancing algorithms in cloud computing environment. International Journal on Cloud Computing: Services and Architecture (IJCCSA), 2(5), pp.1-13.

Saini, H., Upadhyaya, A. and Khandelwal, M.K., 2019. Benefits of Cloud Computing for Business Enterprises: A Review. Available at SSRN 3463631.

Sharma, S., Luhach, A.K. and Sinha, S.A., 2016. An optimal load balancing technique for cloud computing environment using bat algorithm. Indian Journal of Science and Technology, 9(28), pp.1-4.

Shirvani, M.H., Rahmani, A.M. and Sahafi, A., 2018. A survey study on virtual machine migration and server consolidation techniques in DVFS-enabled cloud datacenter: taxonomy and challenges. Journal of King Saud University-Computer and Information Sciences.

Singh, B., Singh, J. and Kumar, S. (2015). Virtualization Techniques and Virtualization Challenges in Cloud Computing: A Review. IJCAT - International Journal of Computing and Technology, 20(6), p.6.

Li, K., Xu, G., Zhao, G., Dong, Y. and Wang, D., 2011, August. Cloud task scheduling based on load balancing ant colony optimization. In 2011 Sixth Annual ChinaGrid Conference (pp. 3-9). IEEE. 